\title{
Beam-Charge Asymmetry associated with DVCS at HERMES
}

F. Ellinghaus ${ }^{\mathrm{a} *}$

(On behalf of the HERMES Collaboration)

${ }^{a}$ DESY Zeuthen, Platanenallee 6, 15738 Zeuthen, Germany

We report the first observation of an azimuthal asymmetry in the hard electroproduction of real photons with respect to the charge of the incoming lepton beam. The asymmetry is attributed to the interference between the Bethe-Heitler process and the deeply-virtual Compton scattering process, which gives access to the latter at the amplitude level. This process appears to be the theoretically cleanest way to access generalized parton distributions. The data have been accumulated by the HERMES experiment at DESY, scattering the HERA 27.6 GeV electron/positron beam off an unpolarized hydrogen gas target.

\section{Introduction}

Inclusive and semi-inclusive deep-inelastic scattering (DIS) is and has been extensively used to study the internal structure of the nucleon. Recent theoretical progress is mostly related to exclusive reactions and their description in terms of generalized parton distributions (GPDs), also referred to as off-forward or skewed parton distributions [1 3]. This theoretical framework takes into account the dynamical correlations between partons of different momenta in the nucleon. The well-known parton distribution functions and form factors turn out to be the limiting cases and moments of GPDs. Of particular interest is the second moment of two unpolarized quark GPDs, which for the first time offers a possibility to determine the total angular momentum carried by the quark in the nucleon [3]. Very recent theoretical ideas indicate that GDPs might be able to describe correlations between the longitudinal and transverse structure of the nucleon [4,5].

\section{Deeply-Virtual Compton Scattering}

The theoretically cleanest way to access GPDs appears to be the deeply-virtual Compton scattering (DVCS) process, i.e. the hard exclusive leptoproduction of real photons with the target nucleon remaining intact. Since the Bethe-Heitler (BH) process has an identical final state and therefore the amplitudes of both processes add coherently, the interference between them can be used to access the DVCS amplitudes. The leading-order and leading-twist interference term [6]

$I= \pm \frac{4 \sqrt{2} m e^{6}}{t Q x_{B j}} \frac{1}{\sqrt{1-x_{B j}}} \times\left[\cos \phi \frac{1}{\sqrt{\epsilon(\epsilon-1)}} \operatorname{Re} \tilde{M}^{1,1}-P_{l} \sin \phi \sqrt{\frac{1+\epsilon}{\epsilon}} \operatorname{Im} \tilde{M}^{1,1}\right]$

*E-mail: Frank.Ellinghaus@desy.de 
depends on the charge and the helicity of the incident lepton, where $+(-)$ denotes a negatively (positively) charged lepton with polarization $P_{l}$. Note that terms suppressed by $O(1 / Q)$ and involving higher $\phi$-moments, were omitted in the above equation. Here $\mathrm{m}$ represents the proton mass, t the square of the four-momentum transfer to the target, $-Q^{2}$ the virtual-photon four-momentum squared and $\epsilon$ is the polarization parameter of the virtual photon. Measuring the dependence of certain cross section asymmetries on the azimuthal angle $\phi$, defined as the angle between the lepton scattering plane and the photon production plane, provides information about the DVCS amplitude combination $\tilde{M}^{1,1}$ which can be expressed in terms of GPDs. The extraction of an asymmetry with respect to the beam spin, accessing the imaginary part of $\tilde{M}^{1,1}$, has already been carried out by the HERMES experiment [7]. The extraction of a beam-charge asymmetry, accessing the real part of $\tilde{M}^{1,1}$, is described in the following.

\section{The Beam-Charge Asymmetry}

The data have been accumulated with the HERMES spectrometer [8] at DESY during the 1998 (2000) running periods. The HERA $27.6 \mathrm{GeV}$ electron (positron) beam was scattered off an unpolarized hydrogen gas target. Events were selected if they contained exactly one photon and one charged track, identified as the scattered electron (positron) and fulfilling the kinematical requirements $Q^{2}>1 \mathrm{GeV}^{2}, W^{2}>4 \mathrm{GeV}^{2}$ and $\nu<23 \mathrm{GeV}$. Here $W$ denotes the photon-nucleon invariant mass and $\nu$ is the virtual-photon energy. The angle between the real and the virtual photon was required to be within 15 and 70 mrad.

In figure 1 the azimuthal dependence of the extracted beam-charge asymmetry (BCA)

$$
A_{C}(\phi)=\frac{N^{+}(\phi)-N^{-}(\phi)}{N^{+}(\phi)+N^{-}(\phi)}
$$

is shown, where $N^{+}\left(N^{-}\right)$represent the luminosity-normalized yields for the corresponding beam charges. Using only events with a missing mass $M_{x}$ between $-1.5 \mathrm{GeV}$ and $+1.7 \mathrm{GeV}$, the data show the expected $\cos \phi$-behavior as can be seen by the solid line

which represents a $P_{1}+P_{2} \cos (\phi)$ fit. The missing mass is defined as $M_{x}=\sqrt{\left(q+P_{p}-k\right)^{2}}$ with $q, P_{p}$ and $k$ being the four-momenta of the virtual photon, the target nucleon and the real photon, respectively. Negative values of the missing mass are due to the finite momentum resolution of the spectrometer, in which case $M_{x}=-\sqrt{-M_{x}^{2}}$ was defined. The beam used at HERMES is polarized; to minimize its effects, positron (electron) data sets were selected to have opposite polarizations $P_{l}\left(-P_{l}\right)$ to eliminate the $\sin \phi$-dependence in the numerator of equation (2). This dependence still exists in the denominator but should be negligible compared to the size of the $\mathrm{BH}$ amplitudes. The fit reveals a positive $\cos \phi$ amplitude with a value of $0.11 \pm 0.04$ (stat) and an offset of $P_{1}=-0.05 \pm 0.03$ (stat). The latter value could possibly be related to a constant term in the interference, omitted in equation (11) since it appears at twist-3 [9]. However, the sign and the size of the amplitude might already contain some valuable information as can be seen by a comparison to model calculations [10] which were carried out in a kinematic regime $\left(<Q^{2}>\right.$ $\left.=2.5 \mathrm{GeV}^{2},<x_{B j}>=0.11,<t>=-0.25 \mathrm{GeV}^{2}\right)$ close to the HERMES kinematics $\left(<Q^{2}>=2.8 \mathrm{GeV}^{2},\left\langle x_{B j}>=0.12,<t>=-0.27 \mathrm{GeV}^{2}\right)\right.$. The calculations are the 


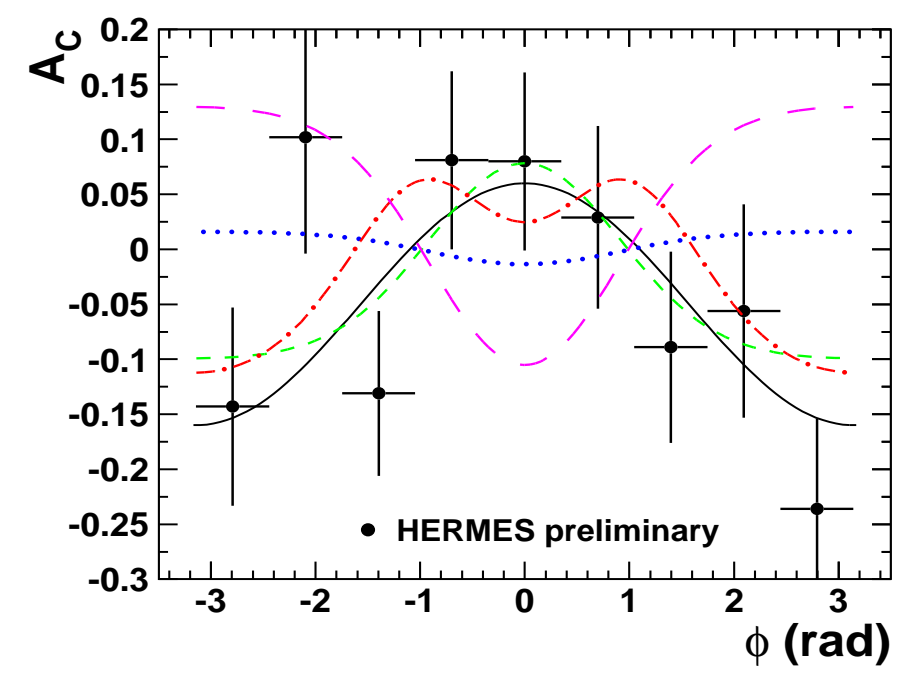

Figure 1. Beam-charge asymmetry for the hard electroproduction of photons as a function of the azimuthal angle $\phi$. The data correspond to the missing mass region between $-1.5 \mathrm{GeV}$ and $+1.7 \mathrm{GeV}$. The solid line represents a $P_{1}+P_{2} \cos (\phi)$ fit to the data. The other curves are model calculations [10] described in the text.

same as described in reference [11]. Certain GPDs are parameterized assuming that the tdependent part factorizes, while the t-independent part is modelled by a two-component form, using the so-called double-distribution formalism [12] completed by the D-term [13]. The D-term is related to the previously unstudied "stress tensor" of hadronic matter. It contributes to the real part of the DVCS amplitude only and therefore can be investigated with the BCA for the first time. Calculating the D-term contribution in the chiral quark soliton model results in the dashed (twist-2) and the dashed-dotted line (twist-3) in figure 11. The twist-2 result without the additional D-term is represented by the dotted line. The long-spaced dashed line is the twist-2 DVCS charge asymmetry when reversing the sign of the D-term. Thus the preliminary HERMES data already favor the existence of the D-term whereby, if it exists, the data clearly determine its sign. However, very recent calculations including twist-3 effects, but using a different GPD model without D-term contribution describe the HERMES data as well [14].

To be able to compare the result in the exclusive region with that in the non-exclusive region, the difference between the $\cos \phi$-weighted moments for either beam charge,

$A_{\mathrm{C}}^{\cos \phi}=\frac{\int_{0}^{2 \pi} d \phi \cos \phi \frac{d \sigma^{+}}{d \phi}}{\int_{0}^{2 \pi} d \phi \frac{d \sigma^{+}}{d \phi}}-\frac{\int_{0}^{2 \pi} d \phi \cos \phi \frac{d \sigma^{-}}{d \phi}}{\int_{0}^{2 \pi} d \phi \frac{d \sigma^{-}}{d \phi}}$

is shown in figure 2 versus the missing mass $M_{x}$. As expected, the asymmetry appears to be non-zero only in the exclusive region, while in the non-exclusive region it is consistent with zero. The asymmetry given by this formula is not due to the real part of the DVCS 


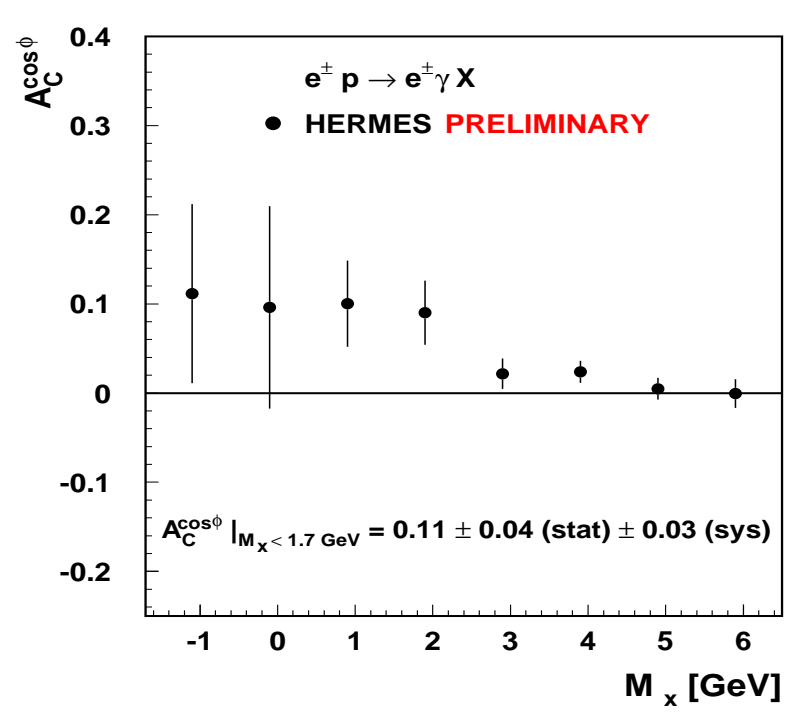

Figure 2. Difference between the positron and the electron $\cos \phi$-weighted moments versus the missing mass $M_{x}$.

amplitudes alone, but has a small modulation from the $\mathrm{BH}$ amplitudes. However, for this preliminary result this method is advantageous due to reduced systematic uncertainties.

In summary, the beam-charge asymmetry in the hard electroproduction of real photons has been measured for the first time. A sizeable asymmetry of $0.11 \pm 0.04$ (stat) \pm 0.03 (sys) has been found in the exclusive region confirming the theoretical expectation.

\section{REFERENCES}

1. D. Müller et al., Fortschr. Phys. 42 (1994) 101

2. A.V. Radyushkin, Phys. Rev. D 56 (1997) 5524

3. X. Ji, Phys. Rev. D 55 (1997) 7114

4. M. Burkardt, Phys. Rev. D 62 (2000) 071503

5. M. Diehl, hep-ph/0205208

6. M. Diehl et al., Phys. Lett. B 411 (1997) 193

7. A. Airapetian et al., Phys. Rev. Lett. 87 (2001) 182001

8. K. Ackerstaff et al., Nucl. Instr. and Meth. A 417 (1998) 230

9. A.V. Belitsky, D. Müller and A. Kirchner, Nucl. Phys. B 629 (2002) 323

10. M. Vanderhaeghen, private communication

11. N. Kivel, M.V. Polyakov and M. Vanderhaeghen, Phys. Rev. D 63 (2001) 114014

12. A.V. Radyushkin, Phys. Rev. D 59 (1999) 014030

13. M.V. Polyakov and C. Weiss, Phys. Rev. D 60 (1999) 114017

14. D. Müller, Proc. of the "European Workshop on the QCD Structure of the Nucleon", April 2002, Ferrara, hep-ph/0206306, to be published in Nucl. Phys. A 